\title{
Acercamiento estadístico a la realidad de los menores infractores en México: legislación y crimen organizado, nuevos desafíos
} Statistical Approach to Reality of Children Offending in Mexico: Legislation and Organized Crime, New Challenges

Recibido: diciembre 5 de 2011 | Revisado: mayo 10 de 2012 | Aceptado: mayo 30 de 2012

\author{
LEÓN DAVID VÁZQUEZ ROMERO*
}

Universidad Iberoamericana, Plantel-Puebla, México

SICI: 2011-2277(201212)11:4<1105:AERMIM>2.0.CO;2-7

Para citar este artículo: Vázquez, L. D. (2012). Acercamiento estadístico a la realidad de los menores infractores en México: legislación y crimen organizado, nuevos desafíos. Universitas Psychologica, 11(4), 1105-1114.

\footnotetext{
Correspondencia Universidad Iberoamericana, Plantel Puebla Blvd. Niño Poblano 2901. Cp. 72197. México, Puebla. Av. Leona Vicario \#705 col. Independencia, Valle de Chalco Solidaridad, Estado de México, México. CP. 56617. E-mail: unleonrojo@ hotmail.com. ResearcherID: Vázquez, L. G-9209. 2012.
}

\section{RESUMEN}

El presente escrito muestra un repaso general de las modificaciones en materia de justicia juvenil en México, para lo cual se abordan de forma somera las distintas escuelas imperantes en el área: penal, asistencialista y garantista, dedicando especial atención al debate actual acerca de encrudecer las penas a la población en cuestión. Se incluye además un análisis estadístico retrospectivo de 1980 a 2007, con lo que al responder a las preguntas: iha cambiado el perfil criminal del menor infractor de los años 80 a nuestros tiempos? y iexiste evidencia que permita suponer que estos cambios y el crimen organizado están relacionados?, se obtuvo con resultados que permiten afirmar que el perfil de los menores infractores mutó con una clara vinculación de delitos contra la salud, homicidio calificado y robo, aunado a una mayor vulnerabilidad a la cooptación por parte del crimen organizado, quedando así abierto el debate sobre la naturaleza del impacto que dichas organizaciones y su oferta de vida tienen en nuestros jóvenes.

\section{Palabras clave autores}

Menores infractores, estadística, legislación mexicana, crimen organizado.

Palabras clave descriptores

Justicia Juvenil, Psicología Social, México.

\section{A B S T R A C T}

This paper presents an overview about modifications in Juvenile Justice in Mexico, for this aim are mentioned main streams in penal, welfares and guarantees areas, putting attention to current discussion on strengthen punishment in the juvenile population. Furthermore, the author present a retrospective statistical analysis between 1980 to 2007, for answer the questions, Has changed the juvenile offender's criminal profile of the 80 's to our times? and Is there evidence suggesting that these changes are related to organized crime?. The results show that the juvenile offender's criminal profile has changed with linkage of crimes against health, murder and theft, coupled with increased vulnerability to cooptation by organized crime, thus leaving open the debate on the nature of the impact that such organizations have and their offer of life for our youth .

Key words authors

Juvenile Offenders, Statistics, Mexican Law, Organized Crime.

Key words plus

Juvenile Justice, Social Psychology, Mexico. 


\section{Legislación, estadística y desafíos: un acercamiento a la realidad de los menores infractores y su relación con el crimen organizado en México}

Al ser México una república, reconoce autonomía a sus estados y permite libertad en el diseño de sus leyes, siempre que se sujeten a una ley máxima ${ }^{1}$, por lo que los códigos estatales, pese a estar sujetos a la ley federal, han legislado en forma distinta el mismo problema. El propósito de este documento es hacer una revisión de los aspectos más relevantes que rodean el tema de las políticas públicas y reportar los datos estadísticos y en materia de menores infractores en el Distrito Federal (DF en adelante).

A modo de antecedente, consideramos importante señalar que, como en muchos otros países, en México se acondicionaron pabellones especiales para menores de edad ${ }^{2}$ en reclusorios para adultos. No fue sino hasta 1965 cuando se crearon edificios especiales para esta población (García, 2007). Es por este tiempo que en la legislación mexicana surgen conceptos tales como tutelar y menores infractores.

Entre 1920 y 1940, se comenzaron a fundar en México los tribunales para menores. De esta forma, los menores quedaron fuera de los juzgados y prisiones de adultos para tener los suyos propios; la tarea de "corregir" hasta ese entonces había recaído mayoritariamente en manos de órdenes religiosas y ahora formaba parte de las tareas del Estado. En 1959, la Organización de las Naciones Unidas (ONU) pronuncia su Declaración de los Derechos del Niño, tras lo cual México adopta tres ejes de intervención: (a) los menores fueron definidos como personas con derecho a una protección especial, (b) se estableció que deberán contar con los apoyos necesarios para desarrollarse de forma saludable y (c) se estipuló que las leyes promulgadas en la materia deberán considerar fundamentalmente el "interés superior del niño" (Trejo, 2006).

Se puede mencionar que han existido tres modelos de intervención en materia de menores in-

1 En el caso de México, esa ley máxima es la Constitución Política de los Estados Unidos Mexicanos.

2 Conocidos como "pericos" en la década de los sesentas. fractores en México: (a) tutelar, (b) de garantías y (c) penal (Trejo, 2006).

\section{Modelo tutelar}

En 1974, surge la Ley para la Creación de los Consejos Tutelares para Menores Infractores en el Distrito Federal y se crea la figura de "consejeros", quienes tenían el propósito de sustituir a los padres del menor y procurar sus necesidades básicas (González, 2006). Con esta ley se pretende pasar de un enfoque penal $^{3}$ a uno de tutela. Sin embargo, unas de las críticas que se le hizo a este modelo es que el Estado, al erigirse como un estado paternalista y atraer la tutela de los adolescentes en cuestión, también arrebata los derechos de los menores.

En ese momento, los menores podían ser detenidos sin órdenes de aprehensión, e incluso sin delitos que perseguir, es decir, con la sola solicitud de los padres. Así es fácil suponer que los jóvenes muchas veces no sabían de qué se les acusaba ni el estado de sus procesos.

\section{Modelo de garantías}

En 1985, la ONU lo declara Año Internacional de la Juventud y en este marco se insta a los gobiernos de distintos países a las "reglas mínimas de las $\mathrm{Na-}$ ciones Unidas para la administración de la justicia de menores", que también se han llamado "reglas de Beijing". Entre otras cosas, se habló de la necesidad de formar profesionales especializados en el tema de menores infractores, quienes paralelamente a satisfacer la administración de justicia, velaran por el respeto a los derechos y bienestar de los niños (Navarro, 2005).

Poco después, en 1989, se lleva a cabo la Convención sobre los Derechos del Niño, la que fue ratificada y promulgada por México. Esta convención acuerda que se reconoce por niño "a todo ser humano menor de dieciocho años de edad, salvo que, en virtud de la ley que le sea aplicable, haya alcanzado antes la mayoría de edad" (Fondo de

3 Entendido como un sistema de penas que hacían judicialmente responsables a los menores. 
Naciones Unidas para la Infancia [UNICEF], 1989). De igual forma, se habla de la necesidad de que, no importando las leyes que se promulguen al respecto, el bienestar del niño será el eje primordial del que se desprenderán todas las políticas públicas; además, se adopta el principio de presunción de inocencia, la obligación de informar al menor de los delitos que se le acusan, y la obligación de ser atendido oportunamente por el órgano judicial competente. De estos acuerdos internacionales surge en México, para el DF, la Ley para el Tratamiento de Menores Infractores en materia común, y para el resto de la república, en materia federal. Esta entra en vigor hacia 1991 y sustituye a la ley de 1974.

Desprendida de este proceso, surge una reforma al artículo cuarto constitucional, que comienza en 1999 y entra en vigor en el 2000, la que tiene por objeto hacer explícito el reconocimiento de los derechos del niño y elevarlos al rango constitucional'. Así mismo, en el 2000 se publica la ley para la protección de los derechos de niñas, niños y adolescentes, entendiéndose por niños a todas (os) los individuos menores de 12 años, y adolescentes a todos (as) los menores de dieciocho años pero mayores de 12 .

\section{Modelo penal}

Expresado en la reforma constitucional del 2005, se deja claro que los menores de doce años que hayan realizado una conducta prevista como delito en la ley, se harán acreedores a rehabilitación y asistencia social; mientas que a los menores de 18 años y mayores de 12 que hayan cometido una conducta antisocial prevista como delito ante la ley, se les aplicará un sistema de justicia integral que vele por sus derechos fundamentales, pero dejando de ser inimputables, dado que el internamiento solo es posible cuando el menor tiene por lo menos 14 años. Esto, como se verá más adelante, es un acierto en materia de derechos de los niños, pero ocasionará polémica a causa de que la reforma no distingue

4 El rango de constitucionalidad garantiza que ninguna ley de las distintas entidades del país podrá desacatar lo establecido por la Constitución. delitos graves, o contra la salud, delitos que irán en incremento (Hernández, 2009).

Hasta antes de la reforma del 2005, no estaban claros los límites de edad para que el Estado pudiera tomar como imputable al menor. Por mencionar algunos ejemplos, en 1998 se establecía como edad mínima para que el Estado pudiera intervenir en el caso de un menor la edad de 6 a 8 años en cuatro entidades, de 9 a 11 años en catorce entidades, de 12 a 14 años en siete entidades, y sin especificar en 7 entidades; mientras que en el caso de la edad máxima para ser considerado menor con 16 años en doce estados, 17 años en 1 estado, 18 años en 18 estados y sin especificar en 1 estado 5 .

Parece ser que de 1980 a 1995 existe una incidencia criminal igual a la que se presentó de 1996 a 2000, sin embargo, habría que recordar que los primeros rangos (de 1980 a 1995) son de cinco años, por lo que la incidencia delictiva es más bien menor que los años posteriores (Figura 1). Respecto al alto número de menores infractores de 1980 a 1985, quizá se podría explicar porque en ese periodo ocurren dos hechos importantes: (a) la devaluación de la moneda en el sexenio del entonces presidente Miguel de la Madrid ${ }^{6}$ (Olivares, 2010) y (b) el temblor de 1985 que destruyó buena parte de la capital del país?

De 1995 al 2000, podemos observar que la incidencia en este rubro es más o menos estable; a partir del 2000 y hasta el 2007, se vislumbra una clara tendencia hacia el incremento de casos de menores que cometen conductas tipificadas por la legislación mexicana como delitos.

Pudiera ser importante mencionar que en México, a partir del 1997, la muerte del narcotraficante Amado Carrillo ${ }^{8}$ alias El señor de los cielos (Silva,

5 Esto queda resuelto con la reforma del 2005 al establecer rangos mínimos de imputabilidad para un menor.

6 Una de las devaluaciones más importantes en la historia de México, se habla de un $3.164 \%$ de devaluación del peso mexicano frente al dólar americano.

7 Dicho temblor es también conocido como "el terremoto de México", con una magnitud de $8.1 \mathrm{MW}$, con una duración aproximada de poco más de dos minutos, y una réplica de gran repercusión. Se calculan 10.000 muertos.

8 Para algunos estudiosos, Amado Carrillo llegó a ser el delincuente más importante de América Latina, cuando recién habían caído los capos colombianos. 


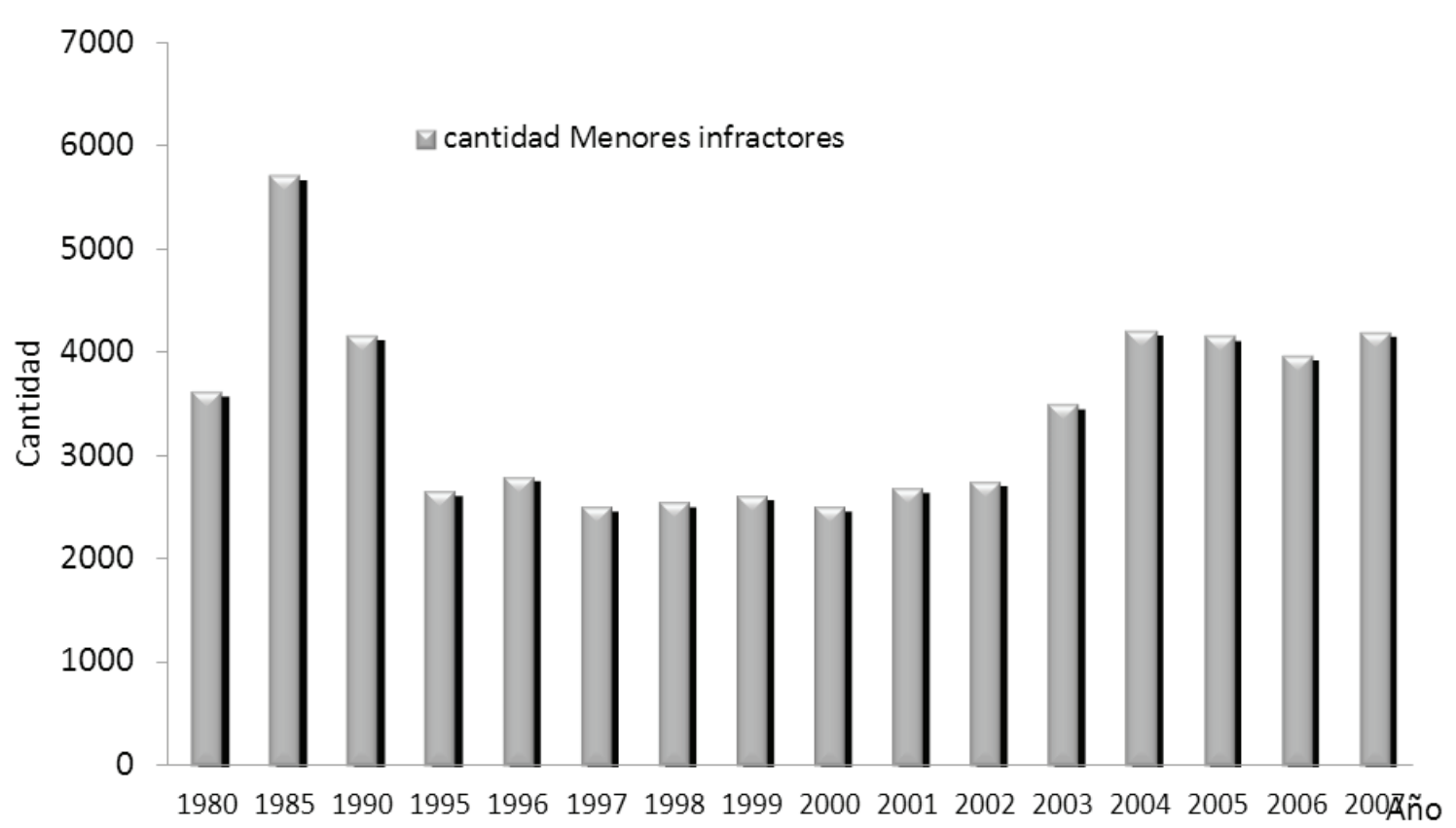

Figura 1. Cantidad de menores infractores de 1980 a 2007 en el Distrito Federal.

Fuente: Los datos fueron extraídos de las citas oficiales del Instituto Nacional de Estadística, Geografía e Informática (INEGI).

1997; Venegas, 1997) dejó entre cárteles dedicados al tráfico de drogas un vacío de poder que desencadenó grandes disputas por el territorio.

La fuga de Joaquín Guzmán Figueroa, alias El chapo de un penal de máxima seguridad, ocurrida en 2001, además de dejar tras de sí serias dudas con respecto a la eficacia del sistema judicial ${ }^{9}$, marcó el inicio de un recrudecimiento de la violencia atribuida al crimen organizado y a su supuesta lucha por la plaza, lo cual hace más marcada la relación entre esta ola de violencia, sin precedentes, y el aumento de ingresos de menores a las instituciones tutelares.

¿En qué ha cambiado el menor infractor de los años ochenta al de nuestros días?, ¿Hay evidencia que permita suponer que el incremento en la inci-

9 Además de dudas sobre si el chapo pagó por su liberación, lo cual no solo puso en entredicho el sistema penitenciario, sino también la transparencia de la administración del entonces recién electo presidente Vicente Fox Quesada. La interrogante de desde dónde viene la complicidad para la fuga del chapo continúa, pues la principal teoría implica al más alto mando del gobierno federal y supone un vínculo de protección de éste último con la organización delictiva de Guzmán (Arista, 2009; Beltrán, 2010; Méndez, 2010; Santos, 2010). dencia de los menores infractores y la guerra entre cárteles de la droga tienen alguna relación?, ¿Qué tipo de delitos son mayoritariamente cometidos por los menores infractores?

De los datos estadísticos que se presentan en las figuras 2 y 3 , se puede extraer que el grueso de la población en cuestión presenta la conducta antisocial en el rango de edad de los 15 a los 17 años, manteniéndose esta tendencia por más de dos décadas, sin presentar cambios drásticos en los últimos años.

Entre las causas de ingreso, de la figura 4 se desprende lo siguiente: el delito de allanamiento de morada no representa un porcentaje significativo en el universo de delitos cometidos por menores infractores, los datos muestran que ha decrecido su incidencia. El delito de daños en propiedad ajena presenta datos importantes, pero nunca llega a constituir el $10 \%$ del total de delitos cometidos, cabe mencionar que este delito tiene un punto álgido en 1985, probablemente relacionado con la devaluación en México (Olivares, 2010); posteriormente, muestra una tendencia a la baja de 1990 a 2000, para luego volver 


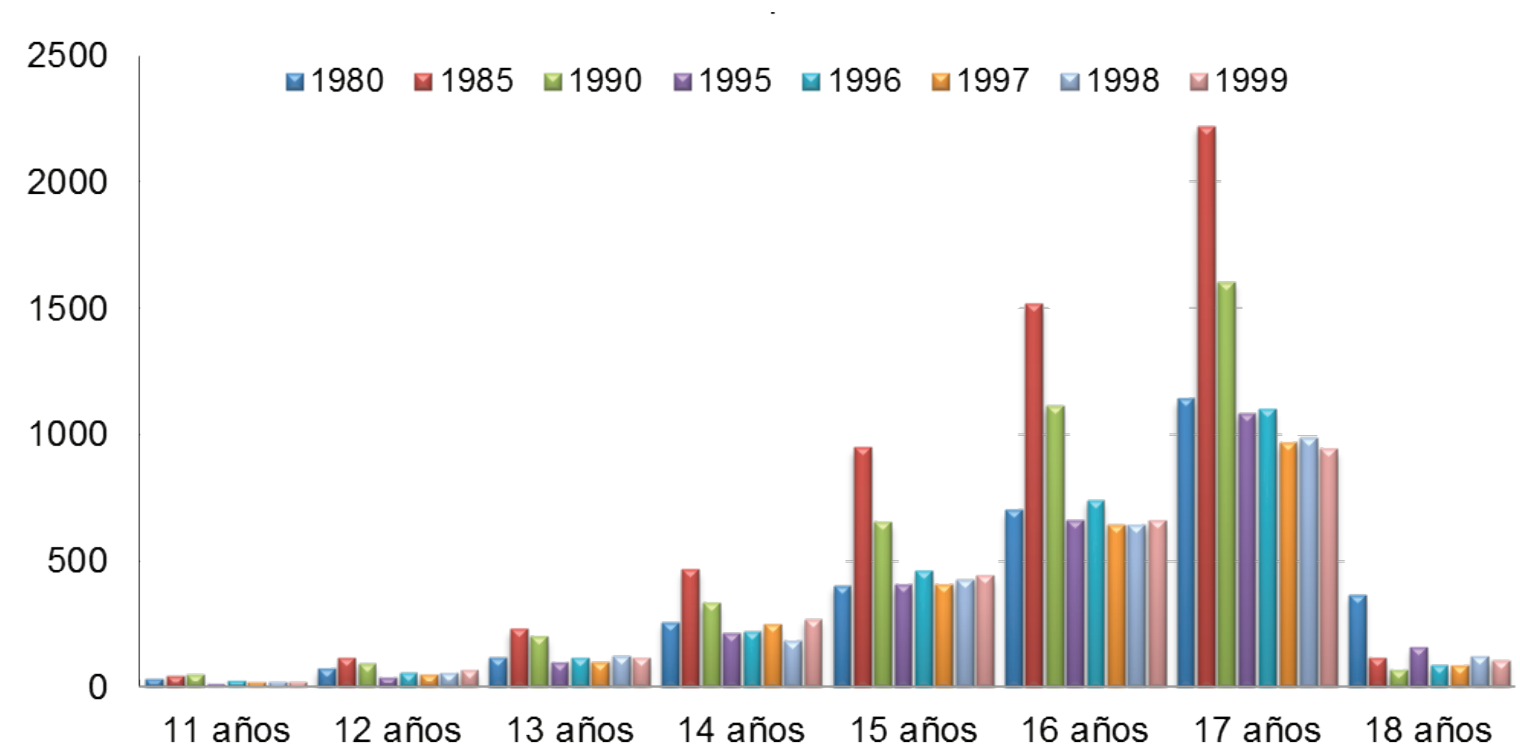

Figura 2. Menores infractores por edad.

Fuente: Los datos fueron extraídos de las citas oficiales del Instituto Nacional de Estadística, Geografía e Informática (INEGI).

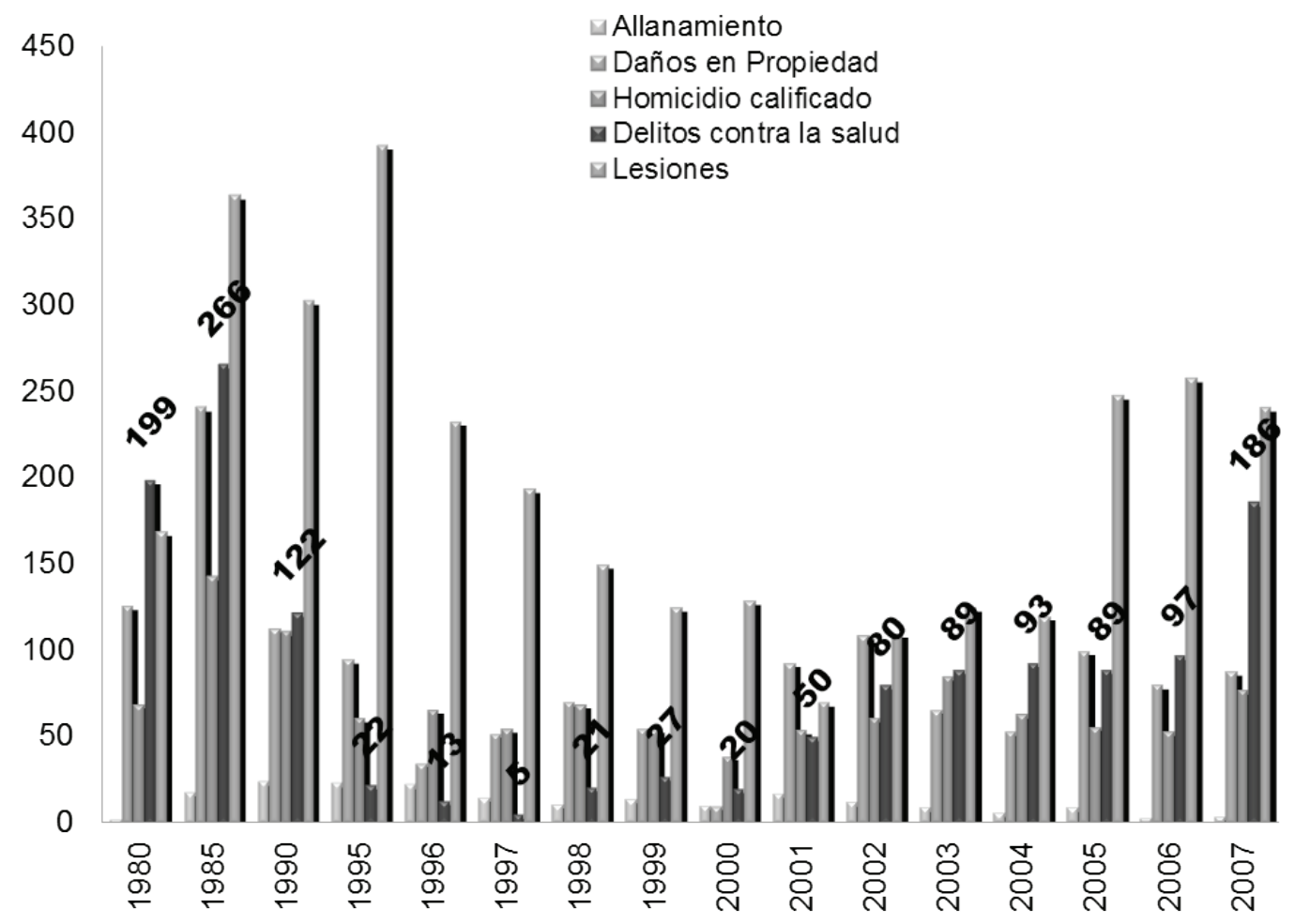

Figura 3. Infractores: ingreso 2000-2007.

Fuente: Los datos fueron extraídos de las citas oficiales del Instituto Nacional de Estadística, Geografía e Informática (INEGI). 


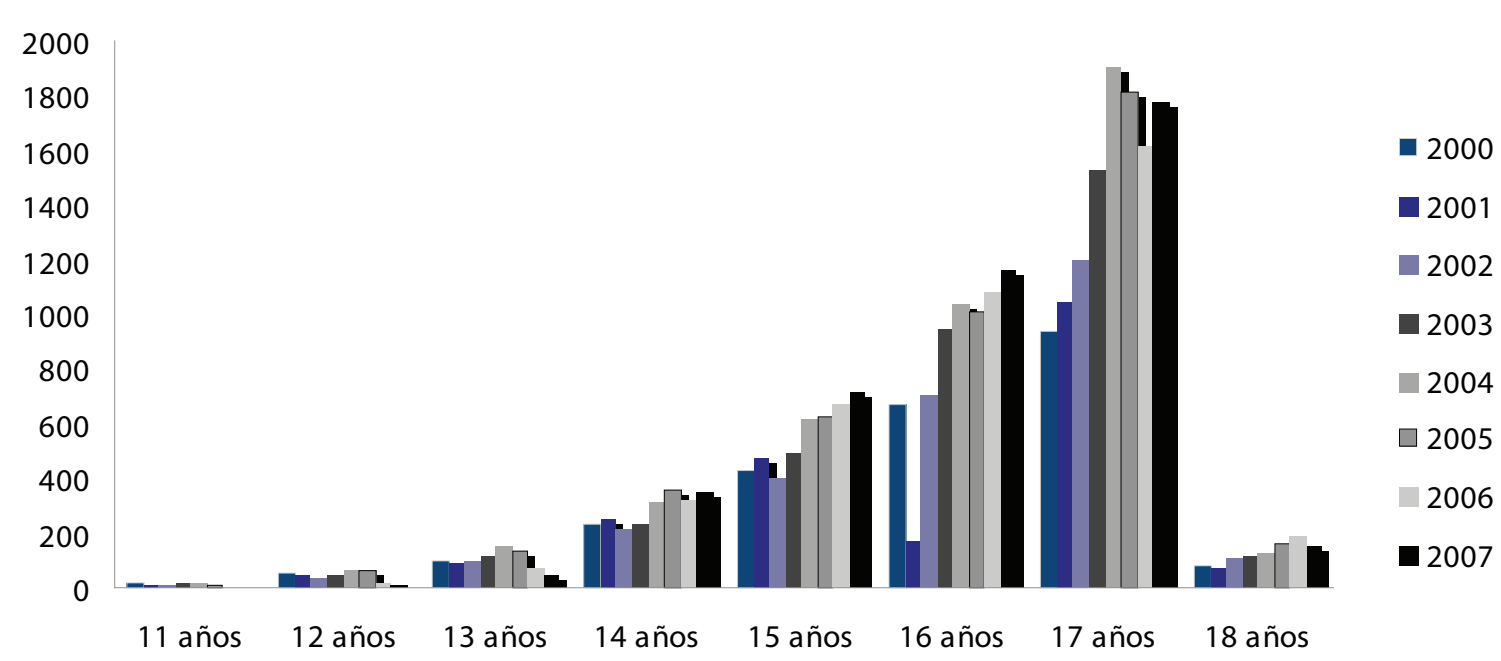

Figura 4. Infractores y tipo de delito.

Fuente: Los datos fueron extraídos de las citas oficiales del Instituto Nacional de Estadística, Geografía e Informática (INEGI).

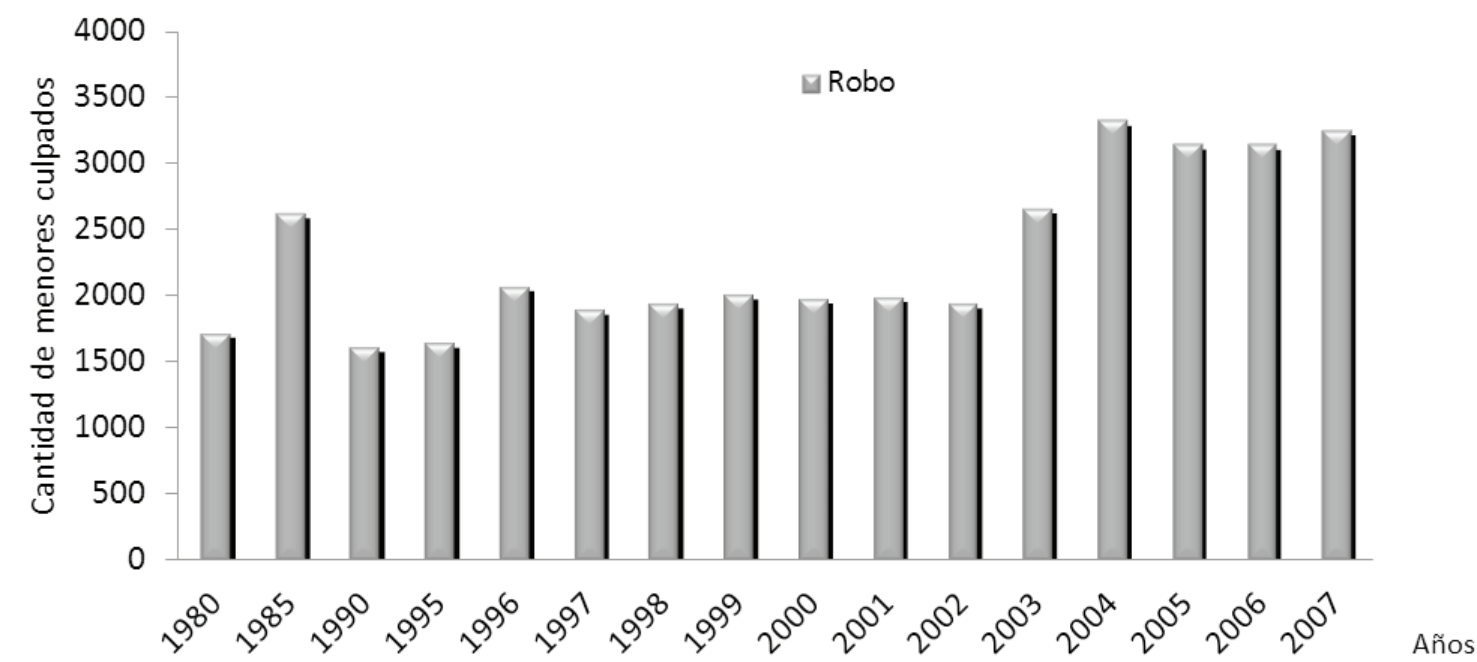

Figura 5. Delito "robo". Menores infractores 1980-2007.

Fuente: Los datos fueron extraídos de las citas oficiales del Instituto Nacional de Estadística, Geografía e Informática (INEGI).

a crecer de forma sostenida de 2000 a 2007. Del delito de homicidio calificado se pueden observar niveles altos en la etapa de la devaluación mexicana del expresidente De la Madrid (Olivares, 2010), y un crecimiento pequeño, pero constante, a partir del año 2000.

Con respecto a los delitos contra la salud asociados al narcotráfico, se puede observar claramente un incremento a partir del año 2000, llegando incluso a sextuplicar la incidencia del 2000 en el 2007. Así mismo, el delito de lesio- nes muestra niveles más altos que en la década pasada, pero no más altos que en la época de la devaluación de 1985.

El delito de privación ilegal de la libertad (Tabla 1), muestra niveles bajos, equiparables a los obtenidos en la década pasada y antepasada. El delito de robo (Figura 5) sigue siendo la conducta criminal mayormente cometida por los jóvenes infractores, mostrando la incidencia más alta de la que se tenga registro, y con una tendencia a incrementarse a partir del año 2002. 
TABLA 1

Privación ilegal de la libertad

\begin{tabular}{cccc}
\hline Año & Casos & Año & Casos \\
\hline 1980 & 5 & 2000 & 4 \\
1985 & 12 & 2001 & 17 \\
1990 & 0 & 2002 & 28 \\
1995 & 33 & 2003 & 5 \\
1996 & 35 & 2004 & 10 \\
1997 & 4 & 2005 & 19 \\
1998 & 8 & 2006 & 8 \\
1999 & 11 & 2007 & 17 \\
\hline
\end{tabular}

Nota. En la presente tabla se muestra el número de menores infractores sentenciados por el delito de "privación ilegal de la libertad" de 1980 a 2007. Cabe señalar que los datos encontrados de 1980 a 1995 se encuentra en intervalos de cinco años, por lo es posible constatar un incremento en los últimos años.

Fuente: Los datos fueron extraídos de las citas oficiales del Instituto Nacional de Estadística, Geografía e Informática (INEGI).

En el rubro de la escolaridad (Figura 6) que muestran los menores infractores, es evidentemente bajo comparado con la edad. Sorprende que desde 1985 a la fecha el índice de escolaridad más común sea el del nivel de secundaria, considerando que la mayor parte de esta población es detenida en el rango de edad de 15 a 18 años. Además, hay una tendencia a incrementar el número de casos con este grado de estudios a partir del 2000.

Todas estas cifras apoyan el concepto de que el fenómeno de los menores infractores en los años ochenta, remitía a la idea de chicos con claros signos de exclusión, como el bajo nivel de estudios y criminalidad vinculada a la falta de oportunidades laborales, educativas y sociales. Cabe preguntar entonces: $i$ Ha cambiado en algo el menor infractor de esos años al menor infractor de nuestros días?

Los números reportados por el Instituto Nacional de Estadística, Geografía e Informática (INEGI) hacen pensar que el grueso de la población en cuestión está en el rango de edad de 15 a 18 años y que, al igual que en el pasado, se mantiene un bajo nivel escolar asociado a la edad de los adolescentes ${ }^{10}$; lo que habla de programas educativos que no han sido eficaces, que no han logrado permear esta población, ni cambiar la tendencia de veinte años a

10 Esto quiere decir que en el rango de 15 a 18 años se esperaría un nivel de estudios por lo menos de preparatoria.

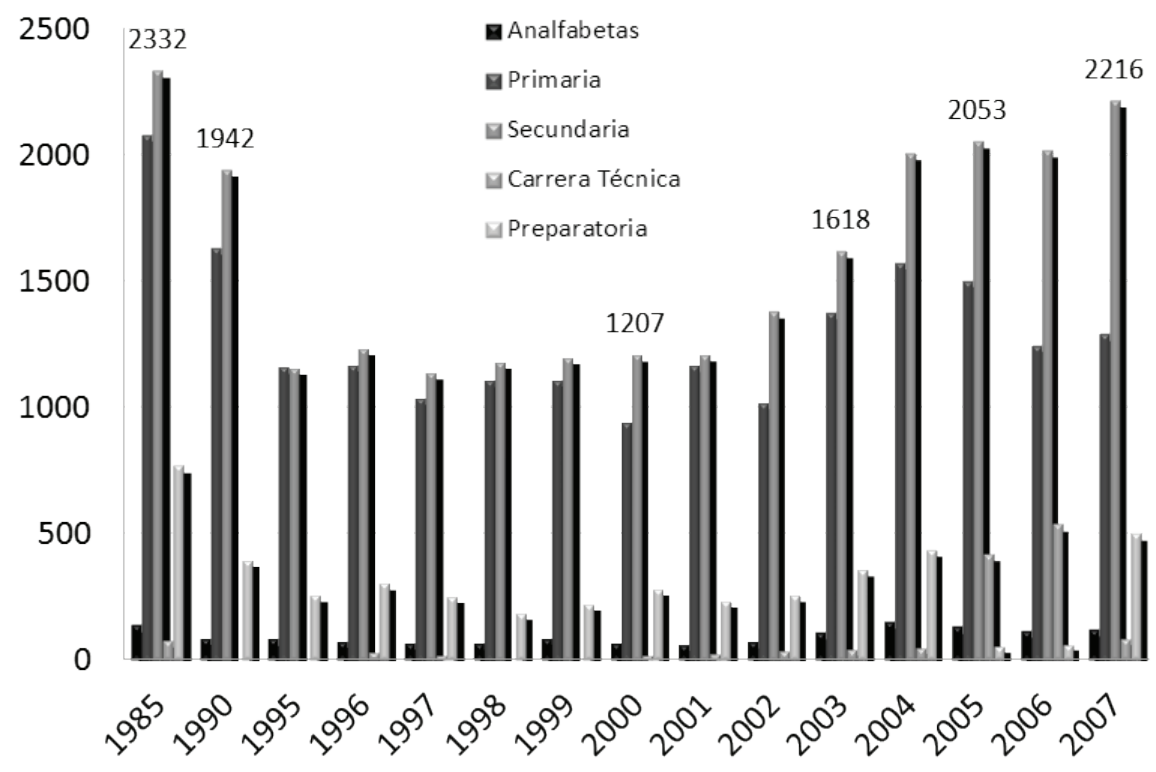

Figura 6. Escolaridad menores infractores.

Fuente: Los datos fueron extraídos de las citas oficiales del Instituto Nacional de Estadística, Geografía e Informática (INEGI). 
la fecha, incluso puede verse un claro incremento en el índice de delitos contra la salud, fuertemente asociados con el narcotráfico, cuyas cifras reportadas por el INEGI se duplicaron de 2006 a 2007, y muestra claros signos de seguir incrementando en años recientes (Covarrubias, 2010). Lo anterior supone un gran problema, toda vez que pareciera que el narcotráfico en México oferta a los jóvenes la posibilidad de iniciar su vida delictiva a edad más temprana (Hernández, 2008), ocasionando esto que los menores tengan una participación cada vez más evidente en puestos medulares, quedando así en evidencia la ausencia de opciones de desarrollo para esta población, por lo que no es difícil pensar que el problema de los menores infractores ahora está asociado al crimen organizado.

Tomando en cuenta el comportamiento de las estadísticas de lesiones y homicidio calificado, se puede ver un claro incremento de este tipo de delitos en los menores, por ello se puede suponer que el menor infractor de nuestros días, en México, es más proclive a la violencia, a involucrarse con el crimen organizado, con un bajo nivel de estudios, con pocas posibilidades educativas, culturales y laborales.

Se ha hablado ya del papel de la legislación en el caso mexicano, de la evolución de las estadísticas y de los cambios presentados por los jóvenes menores infractores. Con todo esto quizá convendría preguntarse: ¿Cuál es el desafío del Estado mexicano en materia de menores infractores?

Quizá al dar una rápida mirada a las figuras, pareciera que los índices delictivos son muy parecidos a los de la década de los ochenta, sin embargo, si se piensa más detenidamente se puede concluir que en esos años México, vivió una de las crisis económicas más importantes de su historia, que había una ley que contabilizaba en las estadísticas a niños en estado de peligro o que habían cometido faltas administrativas, aun así el tipo de delito en esta población cambió.

Los delitos de robo llegaron a cifras nunca antes vistas; eso mismo se puede decir de los delitos contra la salud y las lesiones, el grueso de la edad de los menores infractores (50\%) se mantuvo en el rango de 15 a 18 años. No obstante, su participación en el crimen organizado es cada vez más evidente.
En cuanto al nivel de estudios se aprecia que menos del $15 \%$ alcanza el grado de preparatoria; la mayoría de ellos desertan de la secundaria y se enrolan en actividades tipificadas como delito por las leyes mexicanas. Por ello, es que en abril del 2007 la comisión de Derechos Humanos del Distrito Federal denunció que el $70 \%$ de los menores infractores en el DF, no alcanzaban el grado de secundaria (Galvez, 2007).

El número de menores infractores en México va en aumento. Ante este hecho, las autoridades aventuran nuevas soluciones, como la propuesta en junio del 2010, cuando el procurador de Justicia del Distrito Federal "se pronunció por fijar responsabilidades a los padres de menores infractores que no se limiten a la reparación del daño, sino incluyan la recomposición del tejido social" (Jornada, 2010, p. 35).

Así, el Estado mexicano sepulta de una vez por todas al modelo tutelar y parece que quisiera claudicar del modelo de garantías, para volver nuevamente a un modelo penal. Esto es innegable cuando los estados comienzan a pedir penas cada vez más severas (Álvarez, 201011; Bañuelos, 2008²; Vega, $2010^{13}$ ) como si el solo hecho del castigo ejemplar fuera a ser suficiente para detener el incremento de los delitos.

En esta lógica de los números, podría pensarse que los jóvenes en México decidieron dejar de estudiar e ir por un camino fácil, pero no es así cuando se examinan más detenidamente las cifras que rodean el fenómeno de la juventud en México. En el periodo de 2000 a 2009 se tiene contabilizada la muerte de 1.326 menores en la guerra contra el crimen organizado ${ }^{14}$, según datos de la propia Secretaría de la Defensa Nacional (García, 2010).

11 Edil de León pide endurecer penas contra menores infractores.

12 Aguascalientes aprueba ley en los que los delitos graves para menores infractores podrán tener penas de hasta 20 años de prisión, y tipifica el robo a vivienda como delito grave. Propiciando esto una polémica de la inconstitucionalidad de la ley.

13 El presidente del tribunal superior de Justicia del Distrito Federal pide que se eleven hasta con diez años de cárcel los delitos graves cometidos por menores infractores.

14 Nombre con que se conoce al proyecto nacional en contra del crimen organizado, encabezado por el actual presidente, Felipe Calderón Hinojosa, y que ha tenido como resultado la muerte de más de treinta mil personas en lo que va de su período. 
Quizá valdría la pena preguntarse: ¿Qué opciones de crecimiento real tienen los jóvenes en México actualmente? Según cifras dadas por el actual rector de la Universidad Nacional Autónoma de México, José Narro Robles, en el país existen por lo menos 7.5 millones de jóvenes de entre 12 a 29 años de edad que no estudian ni $\operatorname{trabajan}^{15}$ (García, 2010). Esta gran cifra deja claro la desocupación de un importante sector de la población e invita a formular una nueva interrogante: ¿A qué se dedican estos jóvenes?

La encuesta nacional de la juventud del 2005 menciona que de esa población, al $37.6 \%$ le gustaría seguir en la escuela, $38.4 \%$ preferiría trabajar y al menos $16.9 \%$ ha pensado en irse a trabajar a Estados Unidos (Solano, 2010). Esto quiere decir que no son menores que deseen estar desocupados, más bien son evidencias que apuntan a la exclusión y a la falta de oportunidades educativas y laborales.

La educación media superior y superior en México ha dejado fuera a casi el $80 \%$ de los jóvenes (Solano, 2008), condenándolos a buscar plazas laborales inexistentes y muchas veces engrosando el índice de comercio informal, lo que se puede explicar como una respuesta natural ante el carencia de otras formas de subsistencia.

Tomando en cuenta que paralelo al problema del aumento de la incidencia delictiva en jóvenes, existen problemas de falta de oferta académica y laboral, vale la pena indagar acerca de los montos destinados a dichos sectores. En el 2010, el gobierno mexicano invirtió en materia de seguridad 112.155 millones de pesos, aún con esa cifra se registraron en el país 15.273 homicidios, la cifra más grande en este sexenio, cantidad mayor a la destinada a salud, ciencia y tecnología (103.589 millones de pesos) (Rodríguez, 2011).

Dado el exorbitante presupuesto aprobado anualmente en materia de seguridad, extraña saber que solo en los últimos diez años 1.680 soldados de élite desertaron de las filas castrenses. Militares con entrenamiento táctico avanzado, altamente letal de los que se desconoce su paradero o si están enrolados en el crimen organizado (Michel, 2011).

15 Surge de esto el término tan criticado de "Ninis".
Del 2004 a junio de 2009 van 64.594 soldados que desertan del ejército (Zaragoza, 2009), y no se descarta que muchos de ellos se hayan pasado al lado del crimen organizado.

Dado que la incidencia delictiva en menores infractores se ha elevado aun con el alto presupuesto destinado a seguridad pública, y lejos de debilitar las organizaciones se les está dotando de personal especializado. El desafío que se presenta para México es evaluar en que medida las estrategias contra la delincuencia han rendido los frutos esperados, o quizás, sería mejor recortar dicho presupuesto e invertir ofreciendo a los jóvenes oportunidades académicas y laborales.

\section{Referencias}

Álvarez, X. (2010, marzo 3). Edil de León pide reducir edad penal. El Universal. Disponible en http:// www.eluniversal.com.mx/estados/75047.html

Arista Campo, S. (2009, mayo 16). Hay protección oficial a narcos, acusa el ERPI. La Jornada, p. 36.

Bañuelos, C. (2008, julio 31). Aprueba Aguas Calientes penas más duras contra menores infractores. $\mathrm{La}$ Jornada. Disponible en http://www.jornada.unam. $\mathrm{mx} / 2008 / 07 / 31 /$ index.php?section=estados\&artic le $=038 \mathrm{n} 1$ est

Beltrán, C. (2010, febrero 25). No se protege a "el chapo" ni a nadie, dice Calderón. La Jornada. Disponible en http://www.jornada.unam.mx/2010/02/25/ politica/003n1pol

Covarrubias, A. (2010, diciembre 26). Aumentan menores infractores. El Universal. Disponible en http:// www.eluniversal.com.mx/estados/79066.html

Fondo de Naciones Unidas para la Infancia. (1989). Convención sobre los Derechos del Niño. Recuperado el 5 de agosto, 2011, de http://www.unicef. org/spanish/crc/

Galvez, E. (2007, abril 30). Sin estudios de secundaria, $71.08 \%$ de los menores infractores en el DF en 2006. La Jornada. Disponible en http://www.jornada.unam.mx/2007/04/30/index.php?section $=c$ apital\&article $=048 \mathrm{n} 2 \mathrm{cap}$

García, S. (2007). [Reseña del libro Los menores infractores en México de Ruth Villanueva]. Boletín Mexicano de Derecho Comparado, 40(119), 647-660. 
García, G. C. (2010, marzo 28). Han muerto 1.326 menores en guerra contra el narco. La Jornada, p. 6.

García, L. P. (2010, agosto 26). Necesario, afinar las cifras sobre los "ninis", admite el titular de la S.E.P. La Jornada, p. 35.

González, L. (2006). Justicia penal para adolescentes en conflicto con la ley penal. Iter Criminis. Revista de Ciencias Penales, 3, 89-126.

Hernández, L. G. (2008, octubre 5). Narcotráfico toca puerta a los jóvenes. El Universal. Disponible en http://www.eluniversal.com.mx/ciudad/92126. html

Hernández, S. (2009, noviembre 17). Jóvenes delincuentes se escudan en ley benévola. El Universal. Disponible en http://www.eluniversal.com.mx/ estados/73702.html

Instituto Nacional de Estadística, Geografía e Informática. (2009). Anuario estadístico de los Estados Unidos Mexicanos. México: Autor.

Jornada, L. (2010, junio 17). Tendencias hacia las reformas en materia de menores infractores. $\mathrm{La}$ Jornada, p. 35.

Méndez, A. (2010, septiembre 28). Enfrenta García Luna cascada de acusaciones de diputados. La Jornada, p. 9.

Michel, V. H. (2011, marzo 7). Desertaron en 10 años 1.680 soldados de élite. Milenio.

Navarro, V. (2005). Obligaciones internacionales de México en materia de justicia penal adolescente: las niñas y adolescentes en conflicto con la ley. Una mirada sociológica. México: Secretaría de Relaciones Exteriores.

Olivares, M. A. (2010). Neoliberalismo y autoritarismo: unidad para la crisis. El periodo de Miguel de la
Madrid. Encuentros. Revista Multidisciplinaria de la División de Ciencias Sociales FES Aragón, UNAM. Disponible en http://www.revistaencuentros.com/ wp-content/uploads/2010/09/Neoliberalismo.pdf

Rodríguez, J. I. (2011, enro 30). A seguridad, más presupuesto que a salud, ciencia y tecnología en conjunto. La Jornada, p. 27.

Santos, M. (2010, mayo 22). Calderón debe aclarar ante los mexicanos que no protege a ningún "cártel": AMLO. La Jornada, p. 10.

Silva, C. P. (1997, julio 6). Presunta muerte de Amado Carrillo "el señor de los cielos". La Jornada. Disponible en http://www.jornada.unam.mx/1997/07/06/ capo.html

Solano, L. P. (2008, agosto 7). La falta de espacios en las Universidades condena a los jóvenes a la marginación. La Jornada. Disponible en http://www. jornada.unam.mx/2008/08/07/index.php?section $=$ sociedad $\&$ article $=047 \mathrm{n} 1 \mathrm{soc}$

Solano, L. P. (2010, agosto 22). Los “ninis”, fracaso del estado: especialistas. La Jornada, p. 2.

Trejo, E. A. (2006). Sistema integral de justicia para adolescentes. México: Cámara de Diputados, Centro de Estudios Sociales y de Opinión Pública.

Vega, M. S. (2010, enero 19). Presidente de la TSJDF pide mayor severidad contra menores. La Jornada, p. 32 .

Venegas, J. M. (1997, julio 10). Presunto asesinato de Amado Carrillo. La Jornada. Disponible en http:// www.jornada.unam.mx/1997/07/10/presunto.html

Zaragoza, G. L. (2009, noviembre 8). Se incrementa el número de soldados en los últimos tres años, informa la Sedena. La Jornada, p. 9. 